\section{Index 2009-2010}

\section{Hauptartikel}

NO als Regulator neuronaler Motilität und Regeneration in einfachen Nervensystemen (Gerd Bicker und Michael Stern) 1/09, 4-12

Genetisch codierte optische Sensoren des neuronalen Membranpotenzials: Was sind die Perspektiven für die hochauflösende Messung elektrischer Signale in kortikalen Hirnstrukturen? (Walther Akemann und Thomas Knöpfel) $1 / 09,13-20$

Epigenetik: Nadelöhr neuronaler Erkrankungen? (André Fischer) 1/09, 21-28

Im gemachten Nest - Struktur und Funktionen neuraler Stammzellnischen (Alexander von Holst und Andreas Faissner) 2/09, 44-55

Neuropeptid S: Ein neues Transmittersystem im Gehirn (Kay Jüngling, Thomas Seidenbecher, Jörg Lesting, Rainer K. Reinscheid und HansChristian Pape) 2/09, 56-62

Die modifizierte Amyloid-Hypothese der Alzheimer-Demenz - intraneuronales Abeta induziert Neurodegeneration (Oliver Wirths und Thomas A. Bayer) $3 / 09,76-82$

Die Mobilität der synaptischen Vesikel (Dirk Kamin und Silvio O. Rizzoli) 3/09, 84-92

Spreading depolarization, Tsunami im Hirn (Jens P. Dreier) 4/09, 108-113

Die synaptische Architektur des Zapfen-Endfüßchens (Christian Puller und Silke Haverkamp) 4/09, 114-123

Ereigniskorrelierte Potenziale: Ansatz, Parametrisierung und Analyseverfahren (Patrick Darius Gajewski, Nele Wild-Wall, Sven Hoffmann und Michael Falkenstein) 4/09, 124-129

Das Mittelohr-Cholesteatom: eine Reise zur Aufklärung der Ursache dieser aggressiven Form der chronischen Mittelohrentzündung (Jürgen-Theodor Fränzer und Holger Sudhoff) $1 / 10,141-150$

Das neuronale Netzwerk der Inneren Uhr (Charlotte Helfrich-Förster) 1/10, 151-156

Soziale Signale erschnüffeln: Chemische Kommunikation und das Vomeronasalorgan (Marc Spehr) $1 / 10,157-164$

Schmerzverarbeitung in multisensorischer Umgebung (Marion Höfle, Michael Hauck, Andreas K. Engel und Daniel Senkowski) 2/10, 172-180

Intrazelluläre Ionenhomöostase und deren Beeinträchtigung bei hepatischer Enzephalopathie (Tony Kelly und Christine Rosemarie Rose) 2/10, 181-188

Auf dem Weg zu einer kognitiven Neurowissenschaft intentionalen Handelns und NichtHandelns (Marcel Brass und Simone Kuehn) 2/10, 189-193

Sekundäre Immundefizienz (Immunparalyse) nach Rückenmarkverletzung (Benedikt Brommer,
Marcel A. Kopp, Ines Laginha und Jan M Schwab) 3/10, 208-217

Künstliche Implantate für die Regeneration peripherer Nerven (Isabell Koxholt und Jörg Mey) $3 / 10,218-225$

Signalwandlung und Signalübertragung: Die zwei Seiten eines Fotorezeptors (Andreas Gieß1, Hanna Regus-Leidig und Johann Helmut Brandstätter) 3/10, 226- 234

Ultraschallkommunikation bei Nagern und ihre Bedeutung für Modelle neuropsychiatrischer Erkrankungen (Markus Wöhr und Rainer K.W. Schwarting) 4/10, 248-258

Von Amyloid und Entzündung: was den Muskel chronisch krank macht (Jens Schmidt) 4/10, 259-265

Statistische Verfahren zur Analyse hochdimensionaler neuronaler Messreihen in Bezug auf neurokognitive Vorgänge (Daniel Durstewitz und Emili Balaguer-Ballester) 4/10, 266-276

\section{Artikel des Quartals}

Connectivity-based segregation of the human striatum predicts personality characteristics (Michael X. Cohen, Jan-Christoph, SchoeneBake, Christian E. Elger und Bernd Weber) vorgestellt von Marc Tittgemeyer und Markus Ullsperger, 1/09, 29-30

Functional proteomics identify cornichon proteins as auxiliary subunits of AMPA receptors (Schwenk, J., Harmel, N., Zolles, G., Bildl, W., Kulik, A., Heimrich, B., Chisaka, O., Jonas P, Schulte U, Fakler B. und Klöcker, N.) vorgestellt von Dietmar Schmitz, Stephan Sigrist, Sarah Shoichet, 2/09, 62-63

Brain extracellular matrix affects AMPA receptor lateral mobility and short-term synaptic plasticity (Renato Frischknecht, Martin Heine, David Perrais, Constanze I. Seidenbecher, Daniel Choquet und Eckart D. Gundelfinger) vorgestellt von Michael Frotscher, 3/09, 94-95

Energy-efficient action potentials in hippocampal mossy fibers (Henrik Alle, Arnd Roth und Jörg R. P. Geiger)vorgestellt von Christian Alzheimer, 4/09, 130-131

Formation and maintenance of Alzheimer's disease beta-amyloid plaques in the absence of microglia (Grathwohl, S.A., Kälin, R.E., Bolmont, T., Prokop, S., Winkelmann, G., Kaeser, S.A., Odenthal, J., Radde, R., Eldh, T., Gandy, S., Aguzzi, A., Staufenbiel, M., Mathews, P.M., Wolburg, H., Heppner, F.L. und Jucker, M.) vorgestellt von Michael Heneka, $1 / 10,164-167$

Regulation of Rap2A by the ubiquitin ligase Nedd4-1 controls neurite development (Kawabe, H., Neeb, A., Dimova, K., Young, S.M. Jr., Takeda, M., Katsurabayashi, S., Mitkovski, M., Malakhova, O.A., Zhang, D.E., Umikawa, M., Kariya, K., Goebbels, S., Nave, K.A., Rosenmund, C.. Jahn, O., Rhee, J. und Brose, N.) vorgestellt Andreas Püschel, 2/10, 193-195

Dendritic organization of sensory input to cortical neurons in vivo (Jia, H., Rochefort, N.L., Chen, X. und Konnerth, A.) vorgestellt von Ulf Eysel, $3 / 10,236-237$
Olfactory coding with patterns of response latencies (Stephan Junek, Eugen Kludt, Fred Wolf und Detlev Schild) vorgestellt von Hartwig Spors, 4/10, 277-280

\section{Forschungsföderung/ Institutsvorstellungen}

Forschergruppe 1103: Neurodegeneration und -regeneration bei ZNS-Erkrankungen des Hundes (Wolfgang Baumgärtner, Wolfgang Löscher und Konstantin Wewetzer) 3/09, 96-98

Das Centrum für Schlaganfallforschung Berlin (CSB) (Matthias Endres, Jens P. Dreier, Golo Kronenberg, Andreas Meisel und Ulrich Dirnagl) 4/09, 132-135

Das Hertie-Institut für klinische Hirnforschung - ein Institutsporträt (Michael Madeja) 2/10, 200-201

JARA-BRAIN: Forschungsallianz zwischen der RWTH Aachen und dem Forschungszentrum Jülich (Ilse Trautwein) 3/10, 238-240

SFB 874: Integration und Repräsentation sensorischer Prozesse (Denise Manahan-Vaughan) $4 / 10,282-283$

\section{Glosse}

Neurobiologie des Fußballs - Gedankenspiele zur Fußball-WM (Georg W. Kreutzberg) 2/10, 195-199

\section{Nachrufe}

Hansjürgen Matthies (1925 -2008)(Klaus Reymann, Joachim Schmidt und Tilman Ott) 1/09, $31-32$

Rainer Klinke (1936-2008) (Horst-Werner Korf, Thomas Deller und Herbert Zimmermann) $2 / 09,64$

\section{Portrait}

Johannes Dichgans: Neurologe und Hirnforscher - ein Job für 30-Stunden-Tage? (Rosemarie Grantyn) $1 / 09$, 32-36

\section{Besprechungen/Vorstellungen}

Bernd-Olaf Küppers: Nur Wissen kann Wissen beherrschen: Macht und Verantwortung der Wissenschaft (besprochen von Georg W. Kreutzberg) $1 / 09,41-42$

Wissen-Hörbuch „Hirnforschung 2 - Wie wir denken und entscheiden“" (besprochen von Anja Hoffmann) 2/09, 73-74

Ein Nobelpreisträger als Hollywood-Star - Auf der Suche nach dem Gedächtnis - Der Hirnforscher Eric Kandel (Filmbesprechung von Randolf Menzel) 3/09, 104-105

Lehrbuch mit Personenkult: Neurowissenschaften. Ein grundlegendes Lehrbuch für Biologie (besprochen von Björn Brembs) 3/09, 105-106 
Dein Gehrin - denken, fühlen, handeln - Eindrücke aus einer Ausstellung im Weltkulturerbe „Völklinger Hütte“ (vorgestellt von Joachim W. Deitmer) $1 / 10,168$

Larry R. Squire und Eric Kandel: Vom Geist zum Molekül, and back again - Gedächtnis. Die Natur des Erinnerns (besprochen von Randolf Menzel) 1/10, 169-170

Jascha Rüsseler: Neuropsychologische Therapie. Grundlagen und Praxis der Behandlung kognitiver Störungen bei neurologischen Erkrankungen (besprochen von Ulrike Beinhoff, Nicole Bührsch und Oliver Peters) 2/10, 205-206

John Medina und Sebastian Vogel: Gehirn und Erfolg - 12 Regeln für Schule, Beruf und Alltag (besprochen von Ricarda Scheiner) $3 / 10,245-246$

Manfred Spitzer und Norbert Herschkowitz: Spitzer-Dreiklang (besprochen von Anja Hoffmann) 4/10, 286-287

Michael Madeja: Das kleine Buch vom Gehirn. Ein Reiseführer in ein unbekanntes Land. (besprochen von Johannes Dichgans) 4/10, 287-288

\section{Autoren}

Akemann, Walther 1/09,13-20

Alzheimer, Christian 4/09, 130-131

Balaguer-Ballester, Emili 4/10, 266

Baumgärtner, Wolfgang 3/09, 96-98

Bayer, Thomas A. 3/09, 76-82

Beinhoff, Ulrike 2/10, 205-206

Bicke, Gerd 1/09, 4-12

Brandstätter, Johann Helmut 3/10, 226- 234

Brass, Marcel 2/10, 189-193

Brembs, Björn 3/09, 105-106

Brommer, Benedikt 3/10, 208-217

Bührsch, Nicole 2/10, 205-206

Deitmer, Joachim W. 1/10, 168

Deller, Thomas 2/09, 64

Dichgans, Johannes 4/10, 287-288

Dirnagl, Ulrich 4/09, 132-135

Dreier, Jens P. 4/09, 108-113, 4/09, 132-135

Durstewitz, Daniel 4/10, 266-276

Endres, Matthias 4/09, 132-135

Engel, Andreas K. 2/10, 172-180

Eysel, Ulf 3/10, 236-237

Faissner, Andreas 2/09, 44-55

Falkenstein, Michael 4/09, 124-129

Fischer, André 1/09, 21-28

Fränzer, Jürgen-Theodor 1/10, 141-150

Frotscher, Michael, 3/09, 94-95

Gajewski, Patrick Darius 4/09, 124-129

Gieß1, Andreas 3/10, 226- 234

Grantyn, Rosemarie 1/09, 32-36

Hauck, Michael 2/10, 172-180

Haverkamp, Silke 4/09, 114-123

Helfrich-Förster, Charlotte 1/10, 151-156

Heneka, Michael, 1/10, 164-167

Hoffmann, Anja 2/09, 73-74, 4/2010, 286-287

Hoffmann, Sven 4/09, 124-129

Höfle, Marion 2/10, 172-180

Jüngling, Kay 2/09, 56-62

Kamin, Dirk 3/09, 84-92

Kelly, Tony 2/10, 181-188

Knöpfel, Thomas 1/09,13-20

Kopp, Marcel A. 3/10, 208-217

Korf, Horst-Werner 2/09, 64

Koxholt, Isabell 3/10, 218-225

Kreutzberg, Georg W. 1/09, 41-42

Kronenberg, Golo 4/09, 132-135
Kuehn, Simone 2/10, 189-193

Laginha, Ines 3/10, 208-217

Lesting, Jörg 2/09, 56-62

Löscher, Wolfgang 3/09, 96-98

Madeja, Michael 2/10, 200-201

Manahan-Vaughan, Denise 4/10, 282-283

Meisel, Andreas 4/09, 132-135

Menzel, Randolf 3/09, 104-105, 1/10, 169-170,

Mey, Jörg 3/10, 218-225

Ott, Tilman 1-09, 3132

Pape, Hans-Christian 2/09, 56-62

Peters, Oliver 2/10, 205-206

Puller, Christian 4/09, 114-123

Püschel, Andreas 2/10, 193 - 195

Regus-Leidig, Hanna 3/10, 226- 234

Reinscheid, Rainer K. 2/09, 56-62

Reymann, Klaus 1/09, 3132

Rizzoli, Silvio O. 3/09, 84-92

Rose, Christine Rosemarie 2/10, 181-188

Scheiner, Ricarda, 3/10, 245-246

Schmidt, Jens 4/10, 259-265

Schmidt, Joachim 1/09, 31-32

Schmitz, Dietmar 2/09, 62-63

Schwab, Jan M. 3/10, 208-217

Schwarting, Rainer K.W.) 4/10, 248

Seidenbecher, Thomas 2/09, 56-62

Senkowski, Daniel 2/10, 172-180

Shoichet, Sarah, 2/09, 62-63

Sigrist, Stephan 2/09, 62-63

Spehr Marc 1/10, 157-164

Spors, Hartwig 4/10, 278-280

Stern, Michael 1/09, 4-12

Sudhoff, Holger 1/10, 141-150

Tittgemeyer, Marc 1/09, 29-30

Trautwein, Ilse 3/10, 238-240

Ullsperger, Markus 1/09; 29-30

von Holst, Alexander 2/09, 44-55

Wewetzer, Konstantin 3/09, 96-98

Wild-Wall, Nele 4/09, 124-129

Wirths, Oliver 3/09, 76-82

Wöhr, Markus 4/10, 248-258

Zimmermann, Herbert 2/09, 64

\section{Keywords}

adaptation $3 / 10,226-234$

ammonia $2 / 10,181-188$

amygdale 2/09, 56-62

amyloid 3/09, 76-82

anxiety disorder 2/09, 56-62

artificial implants 3/10, 218-225

astrocyte $2 / 10,181-188$

autophagy 4/10, 259-265

baseline 4/09, 124-129

behavioural neuroscience 4/10, 248-258

behavioural phenotyping 4/10, 248-258

beta-amyloid 4/10, 259-265

biofilm 1/10, 141-150

biomaterials 3/10, 218-225

carbon monoxide $1 / 09,4-12$

cell stress 4/10, 259-265

cGMP $1 / 09,4-12$

chemoreceptor $1 / 10,157-164$

cholesteatoma $1 / 10,141-150$

circadian clock $1 / 10,151-156$

Ci-VSP 1/09,13-20

CNS 3/10, 208-217

communication $4 / 10,248-258$

cone pedicle 4/09, 114-123

connecting cilium 3/10, 226- 234

cortical networks $1 / 09,13-20$

cortical neurons $1 / 09,13-20$

cross-modal $2 / 10,172-180$

cytokeratins $1 / 10,141-150$

cytoskeleton $3 / 09,84-92$

Drosophila $1 / 10,151-156$

EEG 2/10, 172-180 electrospinning $3 / 10,218-225$

emotion $2 / 10,172-180,4 / 10,248-258$

endocytosis 3/09, 84-92

ERP 4/09, 124-129

exocytosis $3 / 09,84-92$

extracellular matrix 2/09, 44-55

fear behavior $2 / 09,56-62$

fluorescence labeling $3 / 09,84-92$

gap junctions 4/09, 114-123

gene trap 2/09, 44-55

glutamate receptors $4 / 09,114-123$

G-Protein-coupled receptor 2/09, 56-62

growth cone $1 / 09,4-12$

homeostatic plasticity $1 / 10,157-164$

immune system 3/10, 208-217

independent component analysis 4/09, 124-129

insect embryo $1 / 09,4-12$

intentional action 2/10, 189-193

intentional inhibition 2/10, 189-193

intersynaptic vesicle movement 3/09, 84-92

intraneuronal A 342 3/09, 76-82

ischemia $4 / 09,108-113$

machine learning 4/10, 266-276

medial prefrontal cortex 2/10, 189-193

MEG 2/10, 172-180

middle ear $1 / 10,141-150$

multiple single-unit recordings 4/10, 266-276

multisensory integration $2 / 10,172-180$

muscle inflammation 4/10, 259-265

myopathy 4/10, 259-265

neural dynamics 4/10, 266-276

neural stem cell $2 / 09,44-55$

neurodegeneration 3/09, 76-82

neurogenesis $2 / 09,44-55$

neurogenic immune $3 / 10,208-217$

neuron $2 / 10,181-188$

neuronal network $1 / 10,151-156$

neuropeptide S 2/09, 56-62

olfaction 1/10, 157-164

pain $2 / 10,172-180$

parallel 4/09, 114-123

PDF $1 / 10,151-156$

period $1 / 10,151-156$

peripheral nerve $3 / 10,218-225$

pH 2/10, 181-188

photoreceptors $3 / 10,226-234$

prefrontal cortex 4/10, 266-276

primate retina $4 / 09,114-123$

processing $4 / 09,114-123$

protein kinase $\mathrm{G} 1 / 09$, 4-12

reference $4 / 09,124-129$

regeneration $3 / 10,218-225$

ribbon synapse 3/10, 226- 234

self control 2/10, 189-193

sensory neurophysiology 1/10, 157-164

signal transduction $3 / 10,226-234$

social behaviour $4 / 10,248-258$

social chemosignals $1 / 10,157-164$

sodium 2/10, 181-188

source localisation 4/09, 124-129

spinal cord injury $3 / 10,208-217$

spinal syndroms $3 / 10,208-217$

spreading depression 4/09, 108-113

spreading ischemia $4 / 09,108-113$

statistics 4/10, 266-276

stem cell niche $2 / 09,44-55$

subarachnoid hemorrhage 4/09, 108-113

suppression 3/10, 208-217

synapse $3 / 09,84-92$

synaptic vesicle $3 / 09,84-92$

T-cell 4/10, 259-265

transgenic mice 3/09, 76-82

treatment 3/09, 76-82

vertebrate retina $3 / 10,226-234$

vesicle cluster 3/09, 84-92

vesicle mobility $3 / 09,84-92$

vesicle recycling $3 / 09,84-92$

voltage imaging $1 / 09,13-20$

voltage-sensitive fluorescent proteins $1 / 09,13-20$

VSFP $1 / 09,13-20$ 


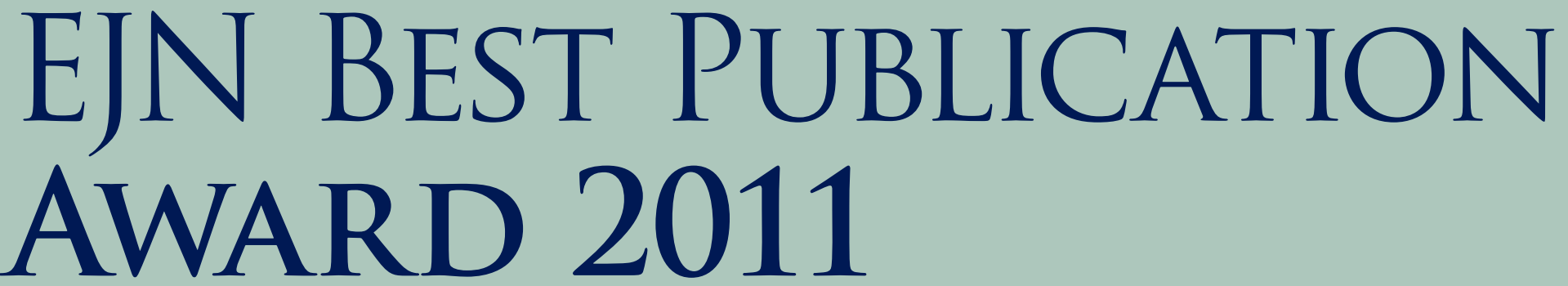

\section{RECOGNIZING THE BEST RESEARCH ARTICLE PUBLISHED IN EJN OVER THE PRECEDING TWO-YEAR PERIOD (PUBLISHED OR ACCEPTED IN YEARS 2009 AND 2010).}

\section{PRIZE: $£ 3,000$ IN COLLABORATION WITH FENS AND WILEY-BLACKWELL}

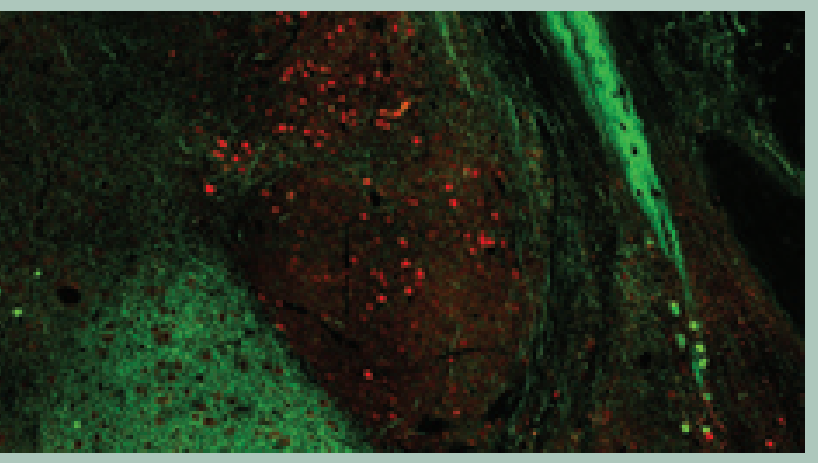

Nominations are accepted from the readership of EJN, including from any author on behalf of the first author of an original article published or accepted in 2009 or 2010. The award will be presented at the FENS Featured Regional Meeting (www.sinapsa.org/snc11) in Ljubljana, Slovenia (September 22-25, 2011).

The award winner will be required to give a Special Lecture at the meeting.

The application consists of the following documents, bundled together into a single PDF file:

- One page summary addressing the significance of the work

- PDF of the article

- Short biosketch (2 pages) of the first author (or first authors in case of equal first co-authorship)

- A signed statement by all authors of the article confirming their support for the nomination and that they agree with the selection of the nominee and, in case of multiple first authors, the selection of the speaker for the Ljubljana meeting.

Applications will be evaluated by a Committee consisting of five Associate Editors and the Editors-in-Chief of EJN.

Applications should be submitted in form of a single PDF attachment to the EJN Editorial Office: editorial.office@ejn.uk.com

Submission deadline: February 28, 2011

www.ejneuroscience.org
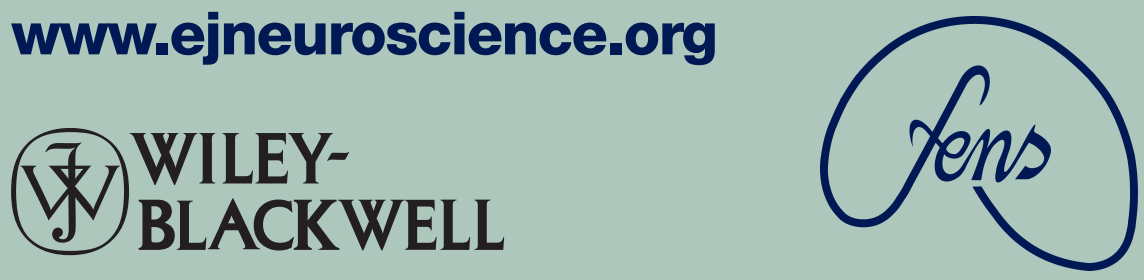


\section{PhenoWorld - Modular Integrated Phenotyping} Neuroscience / Phenotyping / Drug Screening for Mice \& Rats

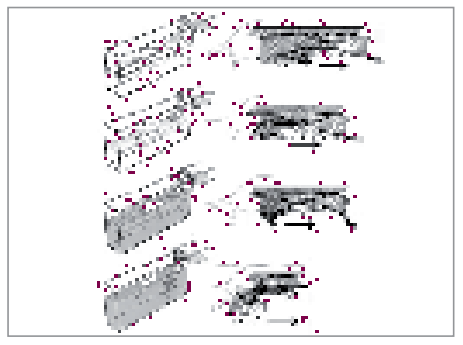

MotoRater

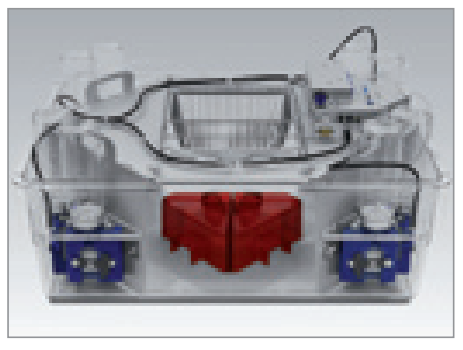

IntelliCage by NewBehavior

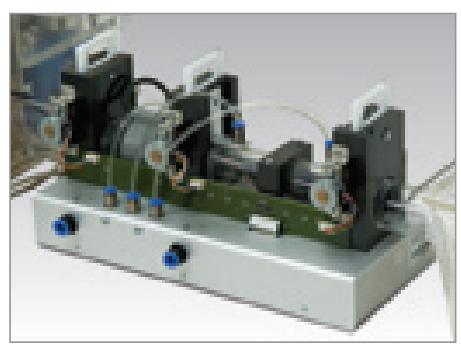

AnimalGate by NewBehavior

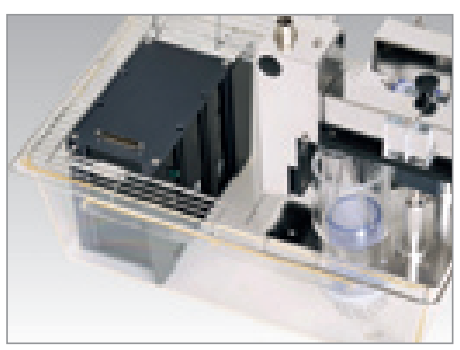

PhenoMaster

\section{NEW Kinematic Analysis}

- MotoRater - standardized modular system for evaluation of locomotor functions using high-speed video tracking: Ladder / Walking / Wading / Swimming

\section{New In Our Product Portfolio 9 NewBehavior}

- Intellicage - fully automated screening for behavioral and cognitive malfunctions of up to 16 mice living in a social group within a home cage Add-ons: AnimalGate / SocialBox

- NeuroLogger - 4 channels wireless EEG \& activity recording

\section{Automated Multi-Dimensional Phenotyping}

- PhenoMaster - automated home cage monitoring for behavioral and / or metabolic phenotyping

- Multi Conditioning - ALL-IN-ONE solution 9 paradigms incl. active \& passive avoidance / latent inhibition / fear conditioning / panic response / place preference / ...

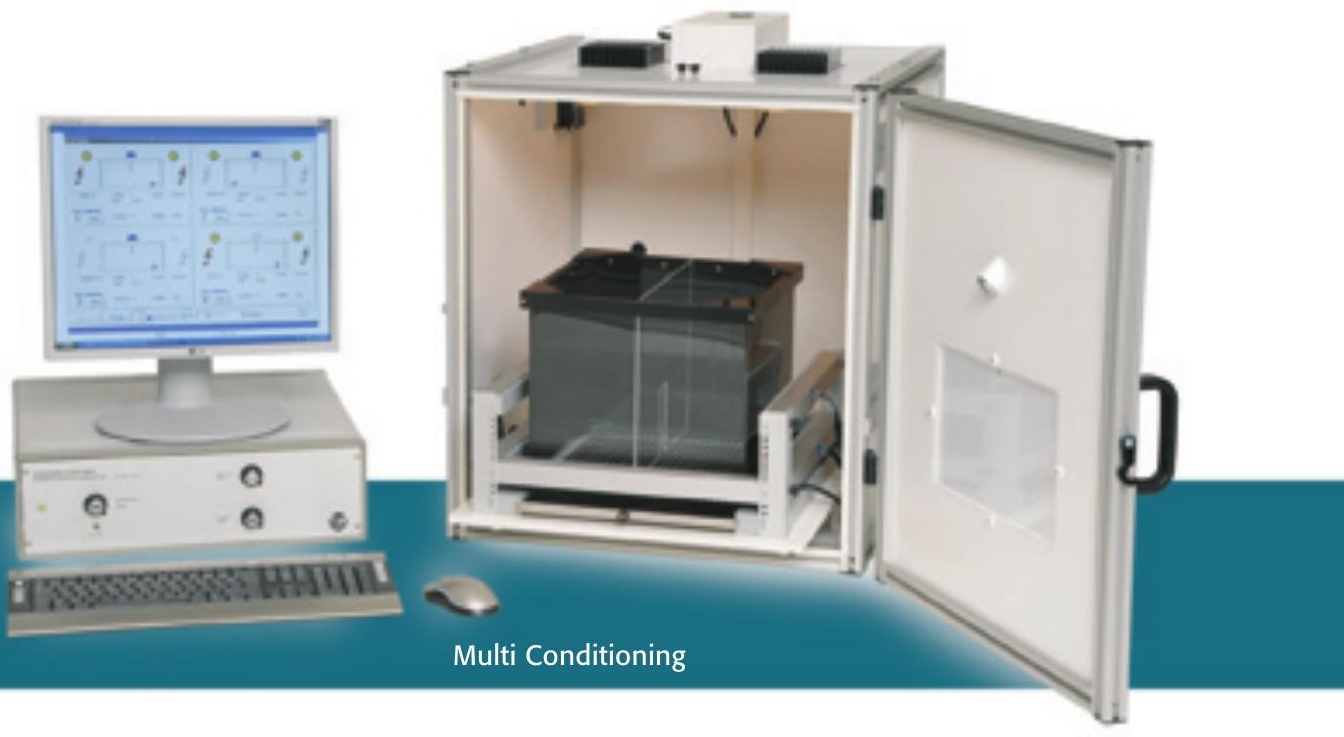

